Original paper

\title{
The role of beam density and arrangement in non-coplanar IMRT exemplified by the irradiation of brain tumors - Parallels to computed tomographic imaging
}

\author{
Gary Razinskas $^{\mathrm{a}, *}$, Tobias Stumm ${ }^{\mathrm{a}, 1}$, Rebekka Kosmala ${ }^{\mathrm{a}}$, Bülent Polat ${ }^{\mathrm{a}}$, Mario Löhr ${ }^{\mathrm{b}}$, \\ Michael Flentje ${ }^{a}$, Klaus Bratengeier ${ }^{a}$
}

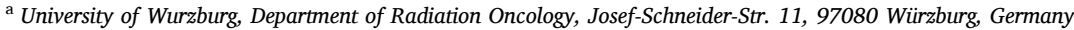

${ }^{\mathrm{b}}$ University of Wurzburg, Department of Neurosurgery, Josef-Schneider-Str. 11, 97080 Würzburg, Germany

\section{A R T I C L E I N F O}

\section{Keywords:}

Non-coplanar intensity modulated

radiotherapy

Brain tumors

Treatment planning study

Computed tomography

\begin{abstract}
A B S T R A C T
Purpose: Parallels between the fields of non-coplanar IMRT and non-coplanar computed tomographic reconstruction are highlighted exemplified by the identification of qualified beam configurations for the irradiation of brain tumors.

Methods and Materials: Four types of beam configurations, i.e. a pure coplanar, a quasi-isotropic and two transitional arrangements, served to systematically examine the impact of parameters such as the sampling rate and the degree of accessibility on plan quality. The resulting set of treatment techniques was compared by means of a Pinnacle ${ }^{3}$ based retrospective planning study on 18 brain tumor cases.

Results and Discussion: A consistent ranking of IMRT beam constellations according to plan quality was established, which directly reflects the necessities of high-quality CT imaging. Once a sufficient dense beam sampling is secured (given by compliance to Nyquist's theorem), the quasi-isotropic (QIso) irradiation produced best treatment plans, followed by a coplanar irradiation complemented by a single orthogonal non-coplanar beam $(\mathrm{CoPl}+1)$. Beams evenly distributed in two orthogonal planes (2-Pl), although using larger portions of the $4 \pi$ space, proved to be less favorable as the beam sequence becomes less dense. The most unfavorable technique is the pure coplanar technique ( $\mathrm{CoPl})$. Generally, techniques with large interbeam distance, i.e. the 2-Pl technique and, to a lesser extent, QIso, are particularly sensitive to a beam number reduction.

Conclusions: Rules established for high quality non-coplanar tomographic imaging are also relevant for noncoplanar IMRT. In this regard, the degree of coverage of $4 \pi$ space is less important than a sufficient dense sampling.
\end{abstract}

\section{Introduction}

In some ways intensity modulated radiotherapy (IMRT) is mathematically connatural to tomographic methods. Parallels between computed tomography (CT) and IMRT were described previously [1]. Bortfeld's considerations regarding IMRT optimization based on the work of Cormack $[2,3]$, a leading pioneer in CT. Therefore, it seems highly advisable to keep established conditions for artifact-free tomographic imaging in mind when examining the quality of beam constellations in radiotherapy - be it IMRT or volumetric arc therapy (VMAT).
In this context, quality shall be seen as the ability to fulfil objectives for a planning target volume (PTV) - organ at risk (OAR) combination.

With the aim to underline such cross-connections, the present work is based on a planning study for a selection of clinical brain tumor cases providing realistic conditions and soundly supported by a summary of results from earlier phantom-based planning studies for non-coplanar IMRT $[4,5]$. While this study focuses on IMRT, some preliminary hypotheses are proposed regarding non-coplanar VMAT, which are subject of future research. Publications on gantry angle or trajectory optimization are omitted in the following unless they help in answering questions

\footnotetext{
* Corresponding author.

E-mail addresses: Razinskas_G@ukw.de (G. Razinskas), t.stumm@uke.de (T. Stumm), Kosmala_R@ukw.de (R. Kosmala), Polat_B@ukw.de (B. Polat), Loehr_M1@ ukw.de (M. Löhr), Flentje_M@ukw.de (M. Flentje), Bratengeier_K@ukw.de (K. Bratengeier).

1 Present address: University of Hamburg-Eppendorf, Department of Anesthesiology, Martinistraße 52, 20246 Hamburg, Germany.
} 
regarding the analogy of CT and intensity modulation in radiotherapy. For the many honorable and advanced approaches of solving such optimization problems the authors refer to a recent review on these topics [6].

This manuscript revolves around the identification of qualified parameters that impact the quality of both CT imaging and non-coplanar IMRT or VMAT and thus elucidates the analogy between the two disciplines. Therefore, in the following we propose two parameters, i.e. the sampling rate and the degree of accessibility, and by systematic variation explore their importance towards improved CT imaging and radiation therapy:

\section{The sampling rate}

According to Nyquist theory the sampling rate plays a key role in computed tomography (Buzug, Eq. 7.67 [7]). In the field of radiotherapy, the sampling rate is represented by the angular distance between neighboring beam axes. Even in coplanar settings an enclosed OAR can be spared - provided that the beam sequence is dense enough [4,5]. Bortfeld [8] pointed out that the number of beams - the sampling rate - can be limited, because every beam is smeared out by finite focal width, scatter phenomena and transmission of leaf tips, resulting in a penumbra of several millimeters. His assessment - based on a decomposition of the beams in Chebyshev polynomials - resulted in a mathematical relation for the required number of equidistant beams $K$, i.e. $K \approx \frac{2 R}{\Delta_{p}}$. Here, $\Delta_{p}=1.4 \Delta$ signifies the deliverable dose peak with the 20\%-80\%-penumbra $\Delta$, while $2 R$ represents the diameter of the target volume including all relevant fine substructures. A descriptive approach is obtained by calculating the number of beams $K$ ' of width $\Delta_{p}$ that can be arranged adjacent to each other on a circular arc of radius $R$, i.e. $K \approx \frac{2 R}{\Delta_{p}} \frac{\pi}{2}$, which is slightly larger than Bortfeld's result. The main message for IMRT planning is that in general only if less than the limiting $K$ or $K$ ' beams are arranged in one plane additional beams enable substantial improvements to the quality of dose distributions by better accomplishing the planning goals. Fenwick and Pardo-Montero [9] followed several approaches to analyze the problem in more detail and developed formulas suggesting up to 2.5 times higher beam numbers than those proposed by Bortfeld. Their radiotherapy-related discussion of the Nyquist criterion is conducted analogously to the CT discussion presented in [7].

Compliance with Nyquist's sampling theorem for radiotherapy by an increase in the total beam number is only reasonable to a certain degree subsequently called the Bortfeld-Fenwick-limit. Below that, an increase of irradiation angles is not expected to result in a substantially improved quality of dose distributions.

2. The degree of accessibility

The theory of tomographic imaging states that full coverage of a semi-shell is not compulsory for good CT imaging. Instead, other conditions (as discussed in 1.) need to be fulfilled.

While dose to healthy tissue in proximity to the PTV is unavoidable in photon radiotherapy, its allocation within the affected healthy tissue can be tailored to some extent. Non-coplanar irradiation allows to redistribute the healthy tissue dose from coplanar sections to sections above or below the PTV thus aiming to reduce dose locally below the damage threshold. As summarized in [6], abundant publications discuss such beam angle optimizations.

Non-coplanar techniques provide a wide range of additional angles of irradiation - far different from the common coplanar directions. This promises to be an attractive way of increasing the likelihood for obtaining suitable irradiation directions that allow for OAR sparing while at the same time avoid disturbing the PTV dose. In the presented work standard coplanar and non-coplanar beam configurations with predefined irradiation directions (i.e. not optimized per individual treatment plan) were investigated. The idea of accessibility relied on increasing the chance to include beams with convenient irradiation directions. Therefore, instead of optimizing angles some authors propose to choose a selection of directions from a pool of possible directions, transferring the optimization problem into a combinatorial problem $[10,11]$.

For fixed beam number IMRT the aforementioned parameters sampling rate and degree of accessibility compete with each other, since usage of the free space of a semi-shell instead of a circle, as suggested by the second parameter, results in larger angular distances and possibly a worse fulfillment of the Bortfeld-Fenwick condition. In other words, the Nyquist limit ought to remain relevant also for non-coplanar IMRT, if the Bortfeld-Fenwick limit is not undershot.

These competing effects were systematically analyzed for noncoplanar IMRT by varying the available number of beams, thereby addressing, among others, the following questions: Are concentrated beam constellations, i.e. small gantry angle differences between neighboring beams, or constellation that take advantage of the scope of $4 \pi$ irradiation favorable? Which circumstances prefer denser sampling or additional zones of irradiation directions?

For this purpose, beam constellations are explored that arrange the available beams into (i) a single plane (coplanar setting, CoPl), (ii) a planar configuration with one additional orthogonal beam $(\mathrm{CoPl}+1)$, (iii) two orthogonal planes (2-Pl) and (iv) a quasi-isotropic irradiation (QIso) (see details in methods section). The usage of the degrees of accessibility (parameter 2 ) provided by non-coplanarity increases independent from the beam number from $\mathrm{CoPl}$ over $\mathrm{CoPl}+1$ and 2-Pl to QIso. Compliance with the Nyquist theorem, however, is expected to be best for $\mathrm{CoPl}$ and $\mathrm{CoPl}+1$, meanwhile it is worse for 2-Pl and QIso; the order of compliance here depends on the number of beams.

The parallels of cone beam CT and VMAT go beyond what has just been described. Although these aspects are not analyzed in this work, they will be presented in the discussion section as an outlook for further research.

Besides beam number and beam arrangement the type of fluence gradation within the present beams is examined as a further parameter. The quality of the dose distribution potentially depends on the number of available segments to form a fluence distribution. In contrast to CT, in radiotherapy dose steps in the order of $1 \%$ of the required dose maximum are sufficient, leading to less demanding requirements regarding the fluence grading. Two approaches were utilized to explore the influence of segment shaping: Firstly, free fluence distribution was allowed by performing a fluence based optimization. Secondly, a segment-based optimization was carried out using two different total segment numbers. These numbers were deliberately kept constant comparing different beam constellations, even for varying beam number, to undermine the argument, that a better plan quality utilizing more beams can be referred to more degrees of freedom in beam forming. It should beconsidered that in this work an increasing number of beams implies a reduction of the available segment numbers per beam.

\section{Methods and material}

This study is geometry based and concentrates on clinical targets instead of the simple geometries investigated in preceding works $[4,5]$. As this manuscript is focused on the medical physics part, the details of patient selection are transferred to the appendix.

\section{Treatment techniques}

There are numerous strategies for daily practice IMRT plan optimization. In order to ensure comparable and reproducible conditions for all considered beam constellations, the techniques applied for patient treatment, i.e. the original clinical plans, were not used for this planning study. Instead, the coplanar reference plans were re-planned using 9 equidistant beams with a maximum of 64 segments aiming at generating 


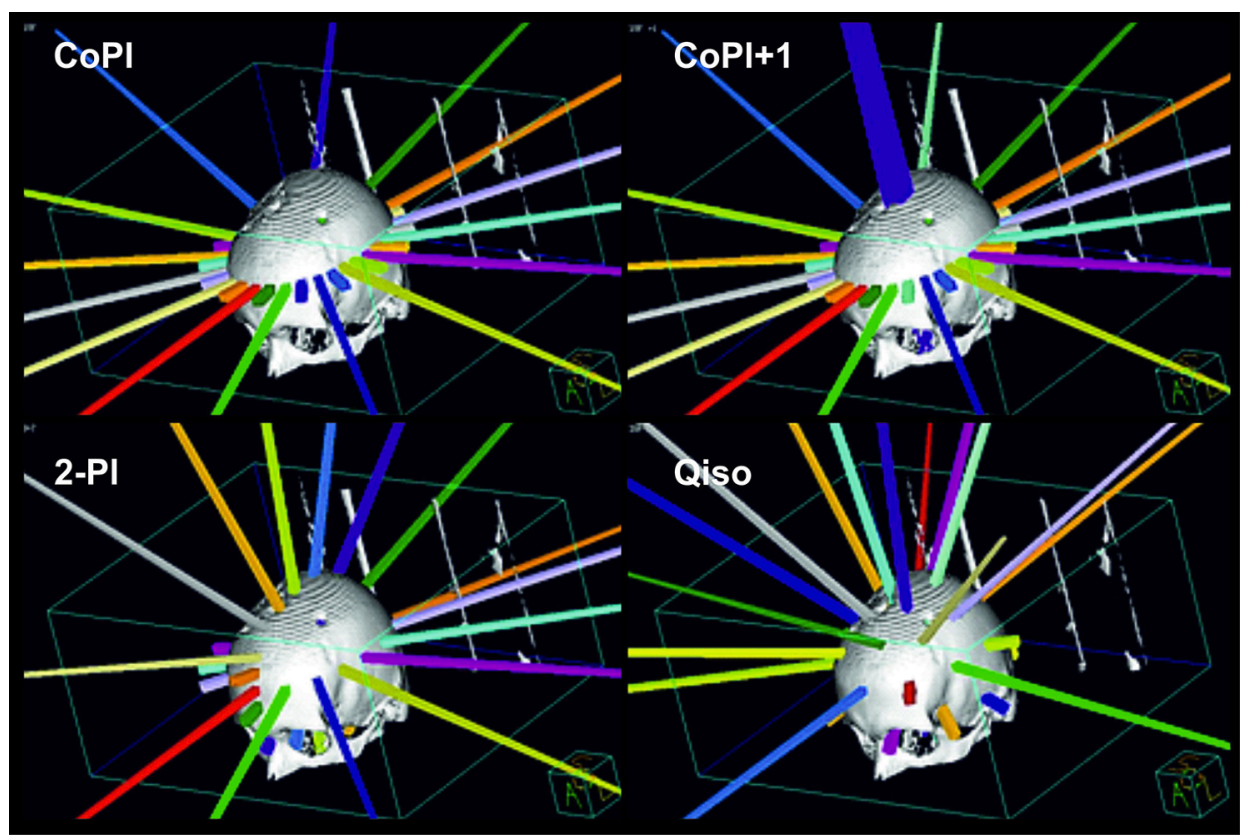

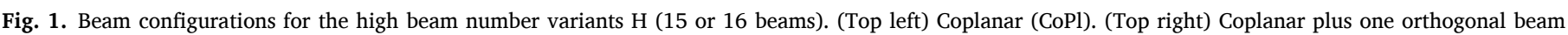

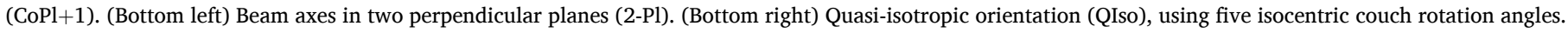

a set of objectives that allows reproducing the course of the dose volume histogram (DVH) of the clinical plans. This procedure guarantees clearly defined patient-related starting conditions for a consistent optimization process of the entirety of each patient's investigated treatment techniques.

Four objectives were defined for each PTV level, two each for the upper and lower limit [12]. Additionally, up to three objectives determined the DVH of each OAR. The set of objectives obtained for the coplanar reference plan served as base for all subsequent plan variants. The optimization process for each variant was identical, which includes that the same number of optimization steps were performed without any manual intervention. The objective function to be minimized was the weighted sum of all relevant quadratic deviations.

Philips $^{\mathrm{TM}}$ Pinnacle $^{3}$ (V 9.2, Philips Radiation Oncology Systems, Fitchburg, WI, USA) was utilized for treatment planning and dose calculation. Plan optimization was carried out by the integrated Raysearch $^{\mathrm{TM}}$ IMRT optimization module (RaySearch Laboratories, Stockholm, Sweden).

\section{Fluence modulation}

Plan optimization was performed employing two different kinds of fluence modification. The first type utilized direct machine parameter optimization (DMPO) and thus simulated real irradiation situations. For each beam configuration two plans were generated by allowing the optimizer to freely allocate a maximum of 64 segments (64S) and 120 segments (120S) to the available beams, respectively. Thereby, segment number dependent trends can be identified.

The second optimization type was based on pure fluence optimization (FO). Unrestricted FO allowed to explore the fundamental properties of certain beam constellations. In contrast to the DMPO process, an efficient approach towards the global minimum is guaranteed by the gradient based FO. Therefore, the result is almost independent from the specific planning system or radiation equipment used.

All plans were optimized under the same conditions: a virtual Elekta Agility head was modeled utilizing $0.5 \mathrm{~cm}$ leaves; the minimum segment width was chosen to be $0.8 \mathrm{~cm}$ (64S) and $0.4 \mathrm{~cm}$ (120S). Single leaf openings were allowed, while the minimum segment area was $4 \mathrm{~cm}^{2}$ and 2 $\mathrm{cm}^{2}$ for $64 \mathrm{~S}$ and $120 \mathrm{~S}$, respectively. Exactly 80 optimization steps were carried out for each plan; the segmented techniques started with 10 optimization steps of fluence optimization, followed by segmentation, 30 steps of segment optimization and a final 40 optimization steps. Monitor unit (MU) data were captured for the DMPO plans (64S and 120S).

\section{Number of beams}

To detect any beam number (n) dependent effects, the number of irradiation axes was varied between

- 9 or 10 axes ( $\mathrm{L}$ - "low beam number"),

- 15 or 16 axes (H - "high beam number"),

respectively. The number of axes within the $\mathrm{L}$ - and $\mathrm{H}$-variants differed by 1 to satisfy high requirements on the symmetry of the beam constellation. For each technique, beam axes were distributed as equidistant as possible under the restrictions of technique generation. No opposing beams were allowed.

\section{Coplanar and non-coplanar techniques}

Four irradiation configurations were studied, namely with increasing non-coplanar characteristics and associated increasing zones of irradiation directions, which contribute to the ability of accessing well suited gantry angles for healthy tissue sparing:

\section{- coplanar $(\mathrm{CoPl})$}

This reference technique was established by an odd number of beams $\mathrm{n}$, which were evenly distributed within one plane. The axis-to-axis distance (the sampling distance) is $20^{\circ}(\mathrm{L})$ and $12^{\circ}(\mathrm{H})$, respectively. - coplanar $+1(\mathrm{CoPl}+1)$

The CoPl configuration was extended by an added orthogonal beam, thus requiring one additional couch position with $90^{\circ}$ couch rotation. The axis-to-axis distance is again $20^{\circ}(\mathrm{L})$ and $12^{\circ}(\mathrm{H})$, respectively.

- two orthogonal planes (2-Pl)

Again, the $\mathrm{n}$ beams from CoPl served as basis. While one "half" of the beams, $(n+1) / 2$, remained in the original position, the other "half", (n-1)/2, was modified by turning the couch to $90^{\circ}$, thus spanning a plane orthogonal to the original one. The beam with zero gantry angle is common to both orthogonal planes. Therefore, both planes 


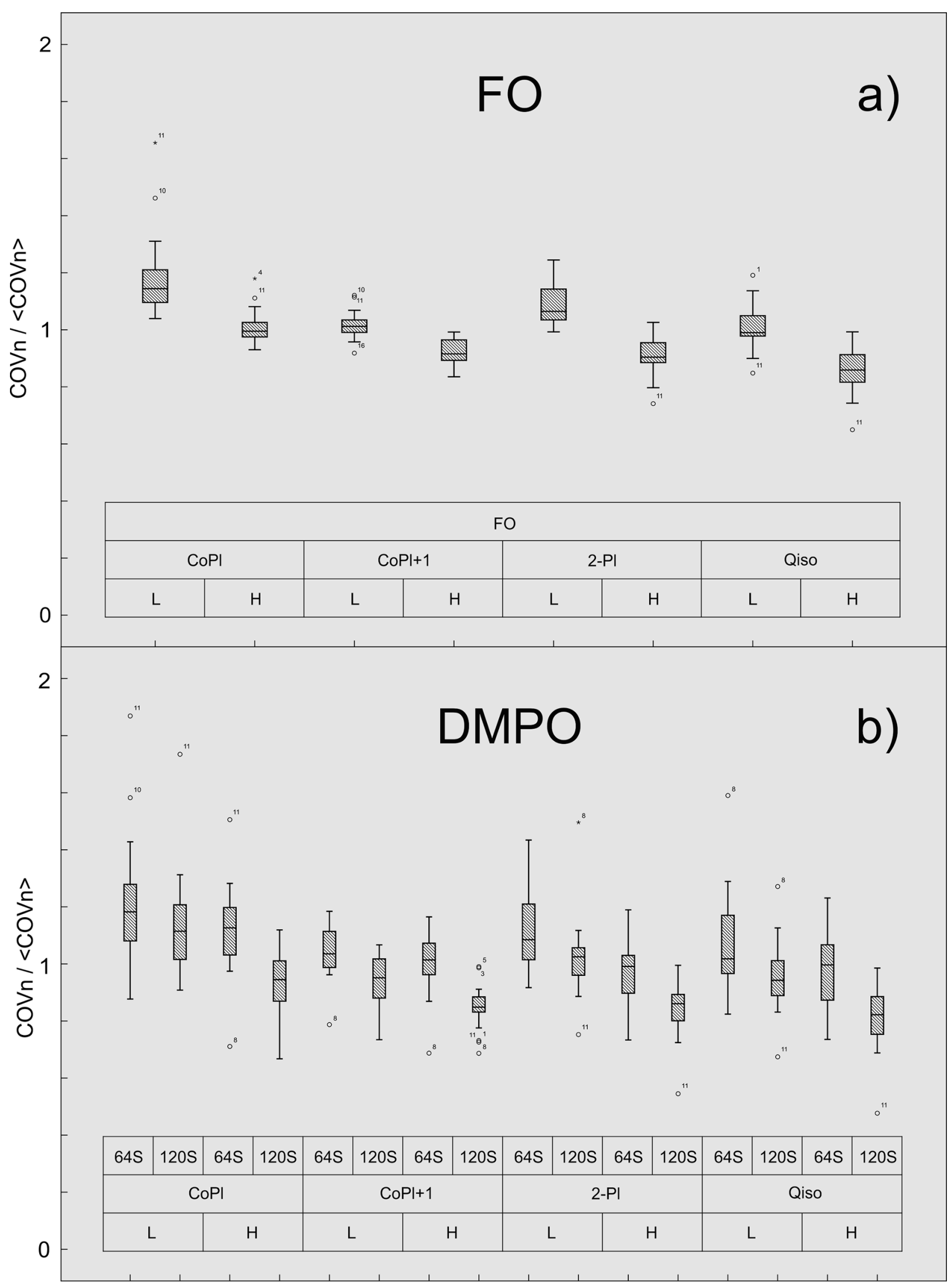

Fig. 2. COV for all FO-based (a) and DMPO-based (b) techniques normalized by the overall mean value. 
Table 1

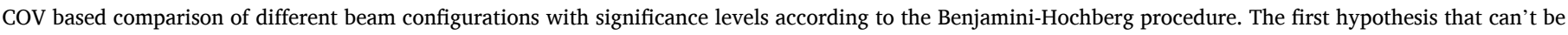
rejected ("techniques are equivalent") is shown in brackets. Preferred techniques in bold are favored avoiding MTF.

\begin{tabular}{|c|c|c|c|c|c|c|c|c|}
\hline & \multirow[b]{2}{*}{ technique 1} & \multirow[b]{2}{*}{ technique 2} & \multicolumn{3}{|c|}{$\mathrm{H}$-variants } & \multicolumn{3}{|c|}{ L-variants } \\
\hline & & & p-value & significance level & preferred technique & p-value & significance level & preferred technique \\
\hline \multirow[t]{6}{*}{ DMPO64S } & CoP1 & $\mathrm{CoPl}+1$ & 0.001 & 0.008 & $\mathrm{CoP} 1+1$ & 0.002 & 0.008 & $\mathrm{CoPl}+1$ \\
\hline & CoPl & 2-P1 & 0.016 & 0.017 & 2-P1 & 0.112 & & \\
\hline & CoPl & QIso & 0.048 & $(0.025)$ & QIso & 0.058 & $(0.017)$ & \\
\hline & $\mathrm{CoPl}+1$ & 2-Pl & 0.472 & & & 0.199 & & \\
\hline & $\mathrm{CoPl}+1$ & QIso & 0.586 & & & 0.845 & & \\
\hline & 2-P1 & QIso & 0.711 & & & 0.170 & & \\
\hline \multirow[t]{6}{*}{ DMPO120S } & CoP1 & $\mathrm{CoPl}+1$ & 0.011 & $(0.008)$ & $\mathrm{CoPl}+1$ & $<0.001$ & 0.008 & $\mathrm{CoPl}+1$ \\
\hline & CoP1 & 2-P1 & 0.022 & & 2-Pl & 0.122 & & \\
\hline & CoP1 & QIso & 0.022 & & QIso & 0.014 & 0.025 & QIso \\
\hline & $\mathrm{CoPl}+1$ & 2-P1 & 0.458 & & & 0.094 & $(0.033)$ & \\
\hline & $\mathrm{CoPl}+1$ & QIso & 0.050 & & QIso & 0.679 & & \\
\hline & 2-P1 & QIso & 0.042 & & QIso & 0.003 & 0.017 & QIso \\
\hline \multirow[t]{6}{*}{ FO } & CoPl & $\mathrm{CoPl}+1$ & $<0.001$ & 0.008 & $\mathrm{CoPl}+1$ & $<0.001$ & 0.008 & CoPl+1 \\
\hline & CoP1 & 2-P1 & $<0.001$ & 0.017 & 2-P1 & 0.048 & $(0.043)$ & 2-Pl \\
\hline & CoP1 & QIso & $<0.001$ & 0.025 & QIso & 0.002 & 0.017 & QIso \\
\hline & $\mathrm{CoPl}+1$ & 2-P1 & 0.306 & $(0.050)$ & & 0.012 & 0.033 & 2-P1 \\
\hline & $\mathrm{CoPl}+1$ & QIso & 0.004 & 0.033 & QIso & 0.948 & & \\
\hline & 2-P1 & QIso & 0.007 & 0.042 & QIso & 0.002 & 0.025 & QIso \\
\hline
\end{tabular}

contained an equidistant and odd number of beams with twice the angular distance between adjacent beam axes compared to CoPl: $40^{\circ}$ (L) and $24^{\circ}(\mathrm{H})$.

- quasi-isotropic (QIso)

This technique is characterized by an even distribution of beams over a hemisphere, thus covering a large solid angle while at the same time maximizing the angular distance between their axes. The beam configurations for both $\mathrm{L}$ - and $\mathrm{H}$-variants are based on platonic solids [13], i.e. on a dodecahedron ( $\mathrm{L}, \mathrm{n}=10$ beams) and on a dodecahedron interlaced with an icosahedron $(\mathrm{H}, \mathrm{n}=16$ beams). The realization of both configurations requires five couch positions separated by $36^{\circ}$. This beam arrangement results in an angular distance between neighboring beams of $42^{\circ}(\mathrm{L})$ and $37^{\circ}(\mathrm{H})$, respectively. If the solid angle portion $\frac{4 \pi}{2 n}$ of each beam axis was transformed to a cone, its apex angle would be $52^{\circ}(\mathrm{L})$ and $41^{\circ}(\mathrm{H})$, respectively.

The investigated beam configurations for the H-variants are depicted in Fig. 1.

\section{Quality indicators}

The weighted sum of quadratic deviations of objectives ("composite objective value", $\mathrm{COV}$ ) can be regarded as a technique's quality criterion, when the set of objectives for each case reliably represents the requirements for the dose distribution. Every relevant PTV is described by two upper and two lower limiting objectives, each OAR by one to three objectives, depending on its distance to the target. For fixed numbers of optimization steps, plans with lower COV can be considered more capable of meeting the needs characterized by the chosen set of objectives. Thus, the COV checks the "elasticity" of each plan to approach the given specifications and marks the inherent potential of a technique. COVn is the normalized COV referring to the reference technique. Such optimized cost functions are common when comparing basic properties of irradiation techniques (see e.g. $[9,10,12]$ ). OAR doses as further quality indicators are described in the additional material.

\section{Statistics}

\section{Wilcoxon rank test}

Pairwise comparisons of techniques have been performed for all 18 patients as follows: $\mathrm{CoPl}$ vs. $\mathrm{CoPl}+1$; $\mathrm{CoPl}$ vs. $2-\mathrm{Pl}$; $\mathrm{CoPl}$ vs. QIso; $\mathrm{CoPl}+1$ vs. 2-Pl; $\mathrm{CoPl}+1$ vs. QIso; and 2-Pl vs. QIso. Every individual comparison was performed for each subtype (FO, S64 and S120 in the L- and Hvariants) separately. As a check a comparison of both L- vs. H-variant and S64 vs. S120 was performed for the entirety of plans.

The Null hypothesis was that the compared techniques (or groups of techniques) do not differ regarding the obtained COV (or OAR sparing, respectively - see appendix). The alternative hypothesis was that they do differ. The statistical evaluation was based on a two-sided Wilcoxon rank test.

\section{Avoidance of multiple testing faults (MTF)}

The data set of each selected technique was compared to the other three techniques. The usual significance level of $p_{s}=0.05$ can be misleading due to the effect of false discovery rates in the case of multiple comparisons [14]. Therefore, the Benjamini-Hochberg procedure [15] was applied as it is more powerful than the Bonferroni-Holm algorithm [16] or the pure Bonferroni algorithm. This procedure was used in a conservative fashion by sorting comparisons of techniques $(\mathrm{m}=6$ in the present case for the COV) according to their p-level starting with the lowest (rank $1, \ldots$, rank $i, \ldots$, rank $\mathrm{m}$ ). Techniques were considered to be of distinguishable quality (rejection of Null hypothesis), as long as plevel $<p_{s} \frac{i}{m}$, starting with $i=1$. Since only three of the six comparisons are independent, this is a rather conservative approach that tends to underestimate the number of rejected Null hypotheses ("no difference between techniques").

\section{Results}

The monitor units for all plans differed by a small amount only: The maximum difference in mean MU per patient never exceeded $+23 \%$ / $-24 \%$. The standard deviation across all plans and cases was only $8 \%$. The mean values over all patients for any technique varied between $+8 \% /-7 \%$ with respect to the mean over all techniques (with a $5 \%$ standard deviation). The groups of $\mathrm{L}$ - and $\mathrm{H}$-variants differed by $<1 \%$, the $120 \mathrm{~S}$ group required $7 \%$ more $\mathrm{MU}$ than the $64 \mathrm{~S}$ group $(\mathrm{p}<0.001$ ).

An overview of all COV values is presented as boxplot diagrams in Fig. $2 \mathrm{a}$ for the FO and Fig. $2 \mathrm{~b}$ for the DMPO (64S and 120S) cases. COV based comparisons of different beam configurations are grouped by segment number and idealized intensity modulation (FO), respectively. Table 1 presents the results of the COV based technique comparison for both the $\mathrm{L}$ - and $\mathrm{H}$-variants. The $\mathrm{p}$-value is derived from a two-sided Wilcoxon rank test. The p-ranking is necessary to apply the termination condition of the Benjamini-Hochberg procedure [15].

In $92 \%$ of all 144 cases plans profit from an increase of segment numbers from 64 to 120 . In addition, in $90 \%$ of all cases the patients would benefit by a beam number increase from $\mathrm{L}$ to $\mathrm{H}$ at constant segment number. The related $\mathrm{p}$-values for pairwise comparisons 


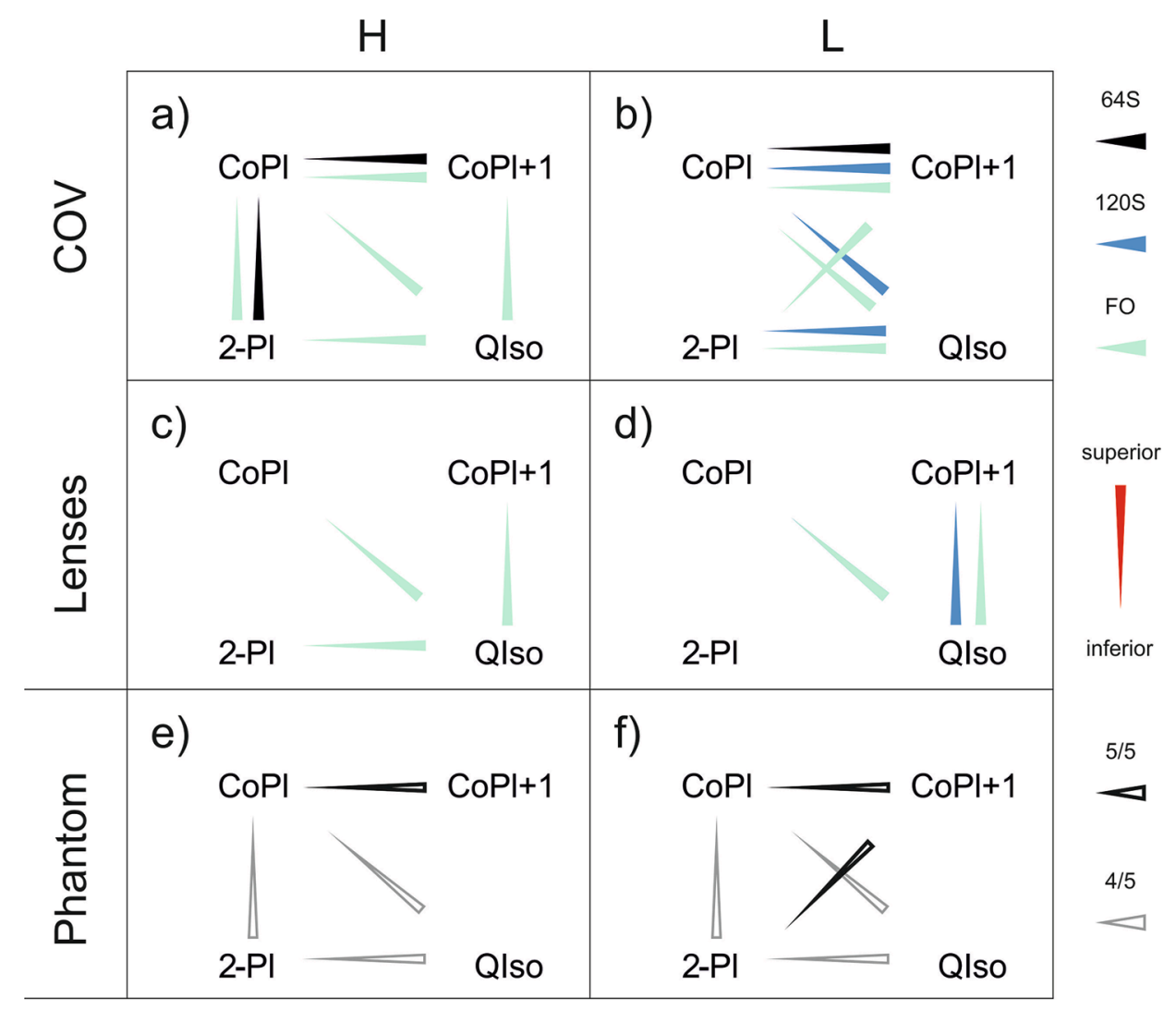

Fig. 3. Significances in the comparison of treatment techniques $\mathrm{CoPl}, \mathrm{CoPl}+1$, 2-Pl, and QIso. Thick ends of triangles point towards better plans. (a,b) Comparison based on COV, an overall plan quality indicator (cf. Table 1). (c,d) Comparison based on mean lens doses (cf. Table A3 and details in the additional material). (e,f) Comparison based on an earlier planning study [4] using a phantom with enclosed simplified geometric PTV and OAR shapes. Results for the $\mathrm{H}$ - and L-variants are shown in the left (a,c,e) and right (b,d,f) column, respectively. 5/5: Superior in 5 out of 5 cases; 4/5: Superior in 4 out of 5 cases. The referring conditions are specified in the main text. according to the Wilcoxon rank test lie below 0.0001 .

\section{Discussion}

Plan quality improvements enabled by an increase in beam number at constant overall number of segments (i.e. constant degrees of freedom) has been demonstrated earlier [17] and is the recipe for the success of the VMAT concept. This fundamental effect was evident for all types of beam constellations investigated in the present study. In addition, a detailed technique comparison sheds light on the concurring effects of accessibility and sampling rate thus enabling more sophisticated interpretations and conclusions. Hence, recommendations for further non-coplanar techniques including VMAT and the definition of future research goals can be derived.

To this end, we include results of a previous phantom-based study [4] to put our statements on a broader basis. There, we referred to the idealized situation of a spherical phantom with a spherical PTV enclosing an OAR. The considered OAR shapes were spherical, cylindrical and bananashaped. The main axis of the latter two were oriented in different ways with respect to the technique's beam constellation. Both the best and the least suitable OAR orientation for each technique were considered for an independent technique comparison. Plan optimization was performed by pure and unrestricted FO allowing to explore the fundamental properties of the aforementioned beam constellations. This resulted in five comparisons of FO. Fig. 3e,f depict the results from the previous study [4]. A technique is interpreted as superior, if it was superior vs. the others in four or five of the five cases ( $4 / 5$ or $5 / 5$, respectively).

\section{Sampling rate vs. Accessibility from non-coplanar directions}

At first glance, a ranking of techniques can be derived from the COV evaluation in the present work (Fig. 3), namely:

$\mathrm{QIso}_{37}>$ ? $\mathrm{CoPl}+1_{12} \approx ? 2-\mathrm{Pl}_{24}>\mathrm{CoPl}_{12}(\mathrm{H})$ with $\mathrm{QIso}_{37}>2-\mathrm{Pl}_{24}$ and

$\mathrm{QIso}_{42} \approx$ ? $\mathrm{CoPl}+1_{20}>2-\mathrm{Pl}_{40}>$ ? $\mathrm{CoPl}_{20}$ (L) with $\mathrm{QIso}_{42}>2-\mathrm{Pl}_{40}$

The indicated numbers mark the angular distance in degrees between neighboring beams. A $>$ B means that technique $\mathrm{A}$ is significantly better than technique $\mathrm{B}, \mathrm{A} \approx$ ? $\mathrm{B}$ expresses that no superiority could be found. A $>$ ? B indicates significant results only in the case of fluence optimization. The obtained order is fully consistent with the outcome obtained by Bratengeier et al. for simple, nevertheless challenging concave PTV shapes [4]. As the present study included much less challenging shapes regarding convex parts of the PTV and steep dose gradients to adjacent OAR structures, the similarity of the obtained rank order is even more remarkable. Differences between the beam constellations are more pronounced for finer fluence modulations as in the case of FO and to a lesser extent in the case of a high number of available segments (120S). While the significance is wiped out for a lower number of segments, the ranking is never inverted.

At second glance, astonishing details referring to the CT-like sampling rate and its limitations as qualitatively predicted by the BortfeldFenwick limit can be extracted, namely:

1. $\mathrm{CoPl}+1$ provides a higher sampling rate and tends to produce better results than 2-Pl, though the latter utilizes a larger solid angle in the non-coplanar regimen. By this, the accessibility should increase in the sense that it becomes more likely to find a gantry angle nearby an "ideal" angle of good choice. The impact on plan quality is more obvious for the L-variants (angular distance between neighboring beams of $20^{\circ}$ vs. $40^{\circ}$ for $\mathrm{CoPl}+1$ vs. $\left.2-\mathrm{Pl}\right)$ than for the $\mathrm{H}$-variants $\left(12^{\circ}\right.$ vs. $24^{\circ}$ ). The sampling rate for the latter is in the order of the Bortfeld-Fenwick limit, which possibly reduces the significance of sampling rate effects and leaves room for improvements by additionally available non-coplanar irradiation angles.

2. QIso is only slightly better than $\mathrm{CoPl}+1$ despite its beams' irradiation directions covering a much wider solid angle. For high beam 
numbers $(\mathrm{H})$, the gained scope becomes relevant, as the disadvantage of a lower sampling rate for QIso decreases when the beam axis distance for $\mathrm{CoPl}+1\left(12^{\circ}\right)$ approaches the Bortfeld-Fenwick limit.

3. In case of low beam numbers (L) 2-Pl even loses its advantage over $\mathrm{CoPl}$ in many cases, although beams in the first, coplanar plane of 2$\mathrm{Pl}$ cover the same angular range than $\mathrm{CoPl}$, albeit with a lower sampling rate. Despite allowing different non-coplanar irradiation directions, beams arranged in the orthogonal plane often cannot compensate for sampling rate related quality losses. For high beam numbers $(\mathrm{H})$, the sampling rate advantage of $\mathrm{CoPl}$ loses strength in relation to 2-Pl as the former approaches the Bortfeld-Fenwick limit.

The behavior of the cost function in the work by Fenwick et al. [9] is similar: Its value is diminished by about $18 \%$ when increasing the beam number from 10 to 20 for a coplanar head and neck plan. In the presented study increasing the beam number from 9 (or 10) to 15 (or 16, respectively) led to reduced $\mathrm{COV}$ values throughout the investigated coplanar and non-coplanar situations (see Fig. 2): CoPl: $0.86 \pm 0.07$, $\mathrm{CoPl}+1: 0.91 \pm 0.07,2$-Pl: $0.83 \pm 0.07$, QIso: $0.85 \pm 0.08$ in the FO case. The values decreased more for both the 2-Pl and QIso techniques that possess beams with larger angular separation. For $\mathrm{CoPl}$ and $\mathrm{CoPl}+1$ the beam number increase plays a minor role, since the Bortfeld-Fenwicklimit is approached for the high beam number $(\mathrm{H})$ case $\left(12^{\circ}\right)$. The mean value over all constellations was $0.86 \pm 0.08$ (FO), $0.85 \pm 0.11$ (120S) and $0.93 \pm 0.10$ (64S). It should be remembered that for segmented techniques a constant number of segments was allowed for the whole plan, independent of the number of beams. Even though fluence shaping might occasionally be insufficient in case of lower segment numbers (64S), increased beam numbers in any case led to better plans.

Strictly speaking, additional zones of irradiation directions should not be equated with additional degrees of freedom, because the beam angles within the zone were not freely chosen; they were as fixed as the beam angles in the coplanar techniques at almost the same number of beams. In a thought experiment, when assigning a cone to each direction of irradiation, the respective direction is responsible for the zone defined by that cone. For an apex angle of about $41^{\circ}(\mathrm{H})$ or $52^{\circ}(\mathrm{L})$ the cones in the QIso and 2-Pl cases begin to touch filling almost the whole solid angle. In contrast, the cones largely overlap for CoPl, leaving a large amount of solid angle blank.

Remarkably, the typical computed tomography effect of sampling rate seems to be more important for overall plan quality than accessibility, allowing for more directions of irradiation within $4 \pi$, as can be seen from the poor performance of the 2-Pl-technique compared to the "almost coplanar" $\mathrm{CoPl}+1$ technique. This point is relevant for static fields, while VMAT inherently offers a dense sampling.

\section{Further expected effects and consequences for VMAT}

As effects known from computed tomography are obviously relevant for radiotherapy, the question arises: are there further conclusions to be drawn or effects to be expected? One aspect would be the "completeness" of the radon space in the sense of fulfilling the Tuy condition [18]. Cone beam CT, which is mathematically connatural with VMAT, in a strict sense cannot completely be reconstructed [7]. In CT its simplified form states that each slice must contain at minimum one ray. This condition is less and less fulfilled the longer the volumes become in cranio-caudal direction. Are the same requirements to be expected in IMRT or VMAT? The simple coplanar cone-beam CT method notably does not enable perfect and complete image reconstruction, instead noncoplanar components are a necessity [7]. The longer the target becomes, the less the Tuy condition is fulfilled. For brain tumors and typical PTV lengths of $10 \mathrm{~cm}$ or less, beams should only be bent by $\arcsin (5 \mathrm{~cm} /$ $100 \mathrm{~cm}) \approx 3^{\circ}$ out of the plane of the coplanar fields. Thus, this effect is expected to play a minor role in the case of brain tumors. In contrast, study of effects related to the Tuy condition could be interesting for VMAT of long targets. Then, for extra-cranial radiotherapy a treatment technique with one table rotation and a small arc might be promising. Some work on non-coplanar VMAT for head and neck tumors is nearing completion.

From the Tuy condition results a second conclusion seems to be selfevident: Regardless of utilizing non-coplanar directions, at minimum one arc should sweep over more than $180^{\circ}+\alpha$, with $\alpha$ being the transversal opening angle of the cone.

\section{Conclusions}

The analysis of coplanar and non-coplanar IMRT irradiationconfigurations in terms of different quality criteria for data of patients with targets in the brain reveals similarities between the fields of non-coplanar IMRT and non-coplanar computed tomographic reconstruction. The usage of a larger space within the $4 \pi$ geometry, as achieved with the different non-coplanar irradiation techniques, not automatically guarantees an improved plan quality. Instead, certain rules established in CT imaging remain valid, first of all the necessity to keep a sufficient dense sampling, even if the whole $4 \pi$ space can be used. Knowledge of the presented competition of beam density and beam arrangement and their influence on the achievable plan quality is of particular significance for the radiation therapy technologists assigned with the task of non-coplanar IMRT dose planning and for the medical physicists that need to be aware of the requirements for good noncoplanar beam configurations and possible limitations. In clinical practice, the established rules allow to tap the full potential of noncoplanar treatment techniques. Moreover, as VMAT intrinsically provides a sufficient dense sampling, the exploration of non-coplanar VMAT is a field of further research.

\section{Additional information}

\section{Use of experimental animals and human participants}

The presented work is a planning study utilizing given CT data of patients irradiated previously and independently from this study. No experiments were conducted on patients. No animals were used.

\section{Ethics approval and consent to participate}

The ethics committee of the University of Würzburg stated no ethical or legal concerns about the evaluation of the listed data.

\section{Declaration of Competing Interest}

The authors declare that they have no known competing financial interests or personal relationships that could have appeared to influence the work reported in this paper.

\section{Additional material}

\section{Patient selection}

Eighteen consecutive adult patients were included in this retrospective planning study. The patients were treated with IMRT due to a complex PTV shape, nearby OARs or a simultaneous integrated boost (SIB), with enclosed PTV at higher dose levels. PTV1, PTV2 and PTV3 denote non-overlapping target volumes with different dose prescriptions. The prescription of non-SIB plans was based on the PTV median dose $\mathrm{D}_{\text {median, }}$ whereas prescription of SIB plans was based on $\mathrm{D}_{95 \%}$ of each PTV level. A summary of the PTV characteristics is given in Table A1. 
Table A1

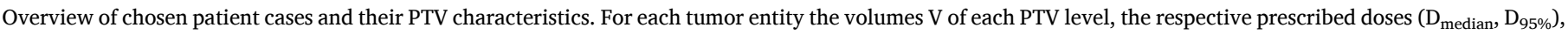
and the number of fractions Fx are listed.

\begin{tabular}{|c|c|c|c|c|c|c|c|c|c|}
\hline & Tumor entity & PTV1 V $\left[\mathrm{cm}^{3}\right]$ & PTV2 V $\left[\mathrm{cm}^{3}\right]$ & PTV3 V $\left[\mathrm{cm}^{3}\right]$ & PTV1 $D_{\text {median }}[\mathrm{Gy}]$ & PTV1 $D_{95 \%}[\mathrm{~Gy}]$ & PTV2 $D_{95 \%}[G y]$ & PTV3 $D_{95 \%}[G y]$ & $\mathrm{Fx}$ \\
\hline 1 & oligodendroglioma & 757 & 83 & 200 & & 48.9 & 54.0 & 56.8 & 27 \\
\hline 2 & glioblastoma & 835 & 115 & & & 45.0 & 55.6 & & 30 \\
\hline 3 & brain metastasis & 18 & 2.6 & & & 52.2 & 55.9 & & 28 \\
\hline 4 & glioblastoma & 425 & 320 & & & 50.9 & 63.9 & & 30 \\
\hline 5 & astrocytoma & 522 & 184 & & & 45.0 & 50.0 & & 25 \\
\hline 6 & macroadenoma & 36 & 10 & & & 47.5 & 49.9 & & 25 \\
\hline 7 & meningeoma & 122 & 39 & & & 49.7 & 59.0 & & 28 \\
\hline 8 & glioblastoma & 108 & 18 & & & 34.6 & 42.5 & & 6 \\
\hline 9 & melanoma metastasis & 102 & 9 & & & 44.9 & 54.8 & & 20 \\
\hline 10 & glioblastoma & 141 & 57 & & & 54.5 & 59.2 & & 30 \\
\hline 11 & astrocytoma & 230 & 116 & & & 48.8 & 54.1 & & 27 \\
\hline 12 & meningeoma & 74 & 15 & & & 51.7 & 52.4 & & 29 \\
\hline 13 & glioblastoma & 67 & 16 & & & 55.6 & 66.7 & & 30 \\
\hline 14 & astrocytoma & 303 & & & 51.8 & & & & 30 \\
\hline 15 & glioblastoma & 84 & & & 28.5 & & & & 6 \\
\hline 16 & glioblastoma & 337 & & & 54.1 & & & & 30 \\
\hline 17 & glioblastoma & 190 & 23 & & & 52.7 & 59.5 & & 30 \\
\hline 18 & glioblastoma & 47 & 27 & & & 28.9 & 29.9 & & 15 \\
\hline
\end{tabular}

\section{Additional quality indicators}

To confirm the results based on the cost function, two groups of OARs were compiled to serve as a second indicator of plan quality. The first, low dose range group contained the lenses of both left and right eye ( $D_{\text {mean }}<4 \mathrm{~Gy}, 25$ relevant OARs), the second, high dose range group referred to parts of the central nervous system, i.e. chiasm $\left(D_{\text {mean }}\right)$, both optic nerves $\left(D_{5 \%}\right)$, medulla oblongata and brain stem $\left(D_{1 \mathrm{~cm}}^{3}\right)$ with dose limits of $40 \mathrm{~Gy}$ or $80 \%$, respectively, for 33 relevant OARs. A particular patient's OAR was only considered as relevant for the evaluation of plan quality, if it was exposed to doses exceeding respective tolerance thresholds at least for one treatment technique. This applies if either an absolute $\left(D_{a}\right)$ or a relative dose $\left(D_{r}\right)$ referring to the prescribed dose to the most central PTV exceeded a dose load of about $20 \%$ below the regime of doses that are potentially critical, i.e. $50 \mathrm{~Gy}$ for the nervous system $[19,20]$, and 5 Gy for the lens in face of short predicted survival times, though a limit of $0.5 \mathrm{~Gy}$ was recently discussed for long-term survival [21]. Organs without dose restrictions during optimization were not considered further.

Notably no transgression of critical dose levels for any OAR threatened. Thus, the comparison of OAR parameters can be considered an "accompanying" aspect, whereas the primary comparison is that of the $\mathrm{COV}$, i.e. a measure representing the entirety of relevant objectives. The comparison was performed for each pair of techniques, for each patient and each objective of an organ recognized as "relevant" in the sense described above. Table A2 lists all OARs taken into account for the evaluation. The two-sided Wilcoxon rank test was applied for all relevant organs and objectives of a group (see below).

\section{OAR based technique comparison}

Two groups of OAR were compared (Table A2), namely parts of the central nervous system (CNS, 41 cases) and lenses (25 cases). In contrast to the COV based technique comparison the results for the OAR based technique comparison (Table A3) were less conclusive. For high beam numbers, significant values (marked bold in Table A3) were obtained only for FO. Here, lower lens doses were achieved by QIso with respect to all other techniques (Fig. 3c). Again, for low beam numbers, QIso showed similar trends towards better lens sparing, even for a segmented situation (120S) (see also Fig. 3d).

The comparison of the L- and $\mathrm{H}$-variants for all four beam constellations resulted in p-values of $0.001,0.020,0.025$, and 0.032 in the Wilcoxon rank test. This implies significant differences in technique quality, if the Benjamini-Hochberg procedure was used at a base of $\mathrm{m}=$ 4. The $\mathrm{H}$-variant was always the preferable one.

For the CNS, only 4 out of the 36 comparisons resulted in p-values below 0.05. At this p-level two accidental false rejections of the null hypothesis were expected. Therefore, no preferences can be deduced since no results were significant according to Benjamini-Hochberg avoidance of MTF.

Table A2

OARs considered in the optimization process by dedicated objectives. Abbreviations: \# - number of considered organs over all 18 patients; $D_{a}$ - absolute dose; $D_{r}$ relative dose.

\begin{tabular}{|c|c|c|c|c|c|}
\hline & & & $\mathrm{D}_{\mathrm{a}}$ & $\mathrm{D}_{\mathrm{r}}$ (alternatively) & $\#$ \\
\hline CNS & medulla oblongata / brainstem & $\mathrm{D}_{1 \mathrm{~cm}}^{3}$ & $40 \mathrm{~Gy}$ & $80 \%$ & 11 \\
\hline \multirow[t]{3}{*}{ (High dose range) } & chiasm & $\mathrm{D}_{\text {mean }}$ & $40 \mathrm{~Gy}$ & $80 \%$ & 8 \\
\hline & left optic nerve & $\mathrm{D}_{5 \%}$ & 40 Gy & $80 \%$ & 5 \\
\hline & right optic nerve & $\mathrm{D}_{5 \%}$ & $40 \mathrm{~Gy}$ & $80 \%$ & 7 \\
\hline Lenses & left lens & $\mathrm{D}_{\text {mean }}$ & 4 Gy & & 12 \\
\hline (Low dose range) & right lens & $\mathrm{D}_{\text {mean }}$ & 4 Gy & & 13 \\
\hline
\end{tabular}


Table A3

OAR based comparison of different beam configurations with significance levels according to the Benjamini-Hochberg procedure. The first hypothesis that can't be rejected ("techniques are equivalent") is shown in brackets. Preferred techniques in bold are favored avoiding MTF.

\begin{tabular}{|c|c|c|c|c|c|c|c|c|}
\hline & \multirow[b]{2}{*}{ technique 1} & \multirow[b]{2}{*}{ technique 2} & \multicolumn{3}{|c|}{$\mathrm{H}$-variants } & \multicolumn{3}{|c|}{ L-variants } \\
\hline & & & p-value & significance level & preferred technique & $\mathrm{p}$-value & significance level & preferred technique \\
\hline \multirow[t]{6}{*}{ DMPO 64S } & CoPl & $\mathrm{CoP} 1+1$ & 0.451 & & & 0.643 & & \\
\hline & CoP1 & 2-P1 & 0.158 & & & 0.560 & & \\
\hline & CoP1 & QIso & 0.013 & & QIso & 0.035 & & QIso \\
\hline & $\mathrm{CoPl}+1$ & 2-P1 & 0.263 & & & 0.214 & & \\
\hline & $\mathrm{CoPl}+1$ & QIso & 0.012 & $(0.008)$ & QIso & 0.019 & $(0.008)$ & QIso \\
\hline & 2-P1 & QIso & 0.031 & & QIso & 0.173 & & \\
\hline \multirow[t]{6}{*}{ DMPO 120S } & CoP1 & $\mathrm{CoP} 1+1$ & 0.189 & & & 0.105 & & \\
\hline & CoP1 & 2-Pl & 0.138 & & & 0.027 & & 2-Pl \\
\hline & CoPl & QIso & 0.044 & & QIso & 0.020 & $(0.017)$ & QIso \\
\hline & $\mathrm{CoPl}+1$ & 2-Pl & 0.263 & & & 0.044 & & 2-Pl \\
\hline & $\mathrm{CoPl}+1$ & QIso & 0.033 & $(0.008)$ & QIso & 0.0081 & 0.0083 & QIso \\
\hline & 2-P1 & QIso & 0.044 & & QIso & 0.173 & & \\
\hline \multirow[t]{6}{*}{ FO } & CoP1 & $\mathrm{CoP} 1+1$ & 0.043 & $(0.033)$ & $\mathrm{CoP} 1+1$ & 0.109 & & \\
\hline & CoP1 & 2-P1 & 0.163 & & & 0.039 & & 2-Pl \\
\hline & CoPl & QIso & 0.009 & 0.017 & QIso & 0.006 & 0.008 & QIso \\
\hline & $\mathrm{CoP} 1+1$ & 2-P1 & 0.562 & & & 0.027 & $(0.025)$ & 2-Pl \\
\hline & $\mathrm{CoPl}+1$ & QIso & 0.020 & 0.025 & QIso & 0.010 & 0.017 & QIso \\
\hline & 2-P1 & QIso & 0.006 & 0.008 & QIso & 0.186 & & \\
\hline
\end{tabular}

\section{Additional results}

The lens dose results are fully compatible with the COV results. Expectedly, the lens dose data were less striking as the optimization was primarily aimed at minimizing the COV. Therein the lens doses were only one aspect besides PTV coverage and sparing of other OARs. These competing goals blur the results of a single OAR.

\section{References}

[1] Bortfeld T, Burkelbach J, Boesecke R, Schlegel W. Methods of image reconstruction from projections applied to conformation radiotherapy. Phys Med Biol 1990;35: 1423-34.

[2] Cormack AM. A problem in rotation therapy with X rays. Int J Radiat Oncol Biol Phys 1987;13(4):623-30.

[3] Cormack AM, Cormack RA. A problem in rotation therapy with X-rays: dose distributions with an axis of symmetry. Int J Radiat Oncol Biol Phys 1987;13: 1921-5.

[4] Bratengeier K, Holubyev K. Characteristics of non-coplanar IMRT in the presence of target-embedded organs at risk. Radiat Oncol 2015;10:207.

[5] Bratengeier K, Seubert B, Holubyev K, Schachner H. Considerations on IMRT for quasi-isotropic non-coplanar irradiation. Phys Med Biol 2012;57(22):7303-15.

[6] Smyth G, Evans PM, Bamber JC, Bedford JL. Recent developments in non-coplanar radiotherapy. Br J Radiol 2019;92(1097):20180908. https://doi.org/10.1259/ bjr.20180908.

[7] Buzug TM. Computed Tomography - from Photon Statistics to Modern Cone-Beam CT. Berlin, Heidelberg: Springer; 2008.

[8] Bortfeld T. The number of beams in IMRT-theoretical investigations and implications for single-arc IMRT. Phys Med Biol 2010;55(1):83-97.
[9] Fenwick JD, Pardo-Montero J. Numbers of beam angles required for near-optimal IMRT: theoretical limits and numerical studies. Med Phys 2011;38(8):4518-30.

[10] Bangert M, Ziegenhein P, Oelfke U. Characterizing the combinatorial beam angle selection problem. Phys Med Biol 2012:57(20):6707-23.

[11] Bangert M, Ziegenhein P, Oelfke U. Comparison of beam angle selection strategies for intracranial IMRT. Med Phys 2013;40(1):011716. https://doi.org/10.1118/ 1.4771932.

[12] Bratengeier K, Meyer J, Flentje M. Pre-segmented 2-Step IMRT with subsequent direct machine parameter optimisation - a planning study. Radiat Oncol 2008;3:38.

[13] Craft D. Local beam angle optimization with linear programming and gradient search. Phys Med Biol 2007;52(7):N127-35.

[14] Benjamini Y. Simultaneous and selective inference: Current successes and future challenges. Biom J 2010;52(6):708-21.

[15] Benjamini, Y, Hochberg, Y. Controlling the False Discovery Rate: A Practical and Powerful Approach to Multiple Testing. Journal of the Royal Statistical Society. Series B (Methodological) 1995;57:12.

[16] Holm S. A Simple Sequentially Rejective Multiple Test Procedure. Scand J Statist 1979;6:6.

[17] Bratengeier K, Gainey MB, Flentje M. Fast IMRT by increasing the beam number and reducing the number of segments. Radiat Oncol 2011;6:170.

[18] Tuy HK. An inversion formula for cone-beam reconstruction SIAM. J Appl Math 1983;43:546-52.

[19] Mayo C, Martel MK, Marks LB, Flickinger J, Nam J, Kirkpatrick J. Radiation dosevolume effects of optic nerves and chiasm. Int J Radiat Oncol Biol Phys 2010;76(3): S28-35.

[20] Mayo C, Yorke E, Merchant TE. Radiation associated brainstem injury. Int J Radiat Oncol Biol Phys 2010;76(3):S36-41.

[21] Thome C, Chambers DB, Hooker AM, Thompson JW, Boreham DR. Deterministic Effects to the Lens of the Eye Following Ionizing Radiation Exposure: is There Evidence to Support a Reduction in Threshold Dose? Health Phys 2018;114: $328-43$. 